\title{
Frequency Influence on the Hot-Electron Noise Reduction in GaAs Operating under Periodic Signals
}

\author{
D. Persano Adorno ${ }^{a}$, M.C. Capizzo ${ }^{a}$ And N. Pizzolato ${ }^{a, b}$ \\ ${ }^{a}$ Dipartimento di Fisica e Tecnologie Relative and CNISM \\ ${ }^{b}$ Group of Interdisciplinary Physics \\ Viale delle Scienze, Ed. 18, 90128 Palermo, Italy
}

\begin{abstract}
A Monte Carlo study of the role of the frequency on the hot-electron intrinsic noise reduction in an $n$-type GaAs bulk driven by two mixed cyclostationary electric fields is presented. Previous numerical results showed the possibility to reduce the diffusion noise under specific wave-mixing conditions. In this work the variations of the noise properties are investigated by computing and integrating the spectral density of the velocity fluctuations. We found that the effect of reduction of the noise level due to the addition of a second field at twice frequency is almost independent of the frequency.
\end{abstract}

PACS numbers: $72.70 .+\mathrm{m}, 05.40 . \mathrm{Ca}, 72.30+\mathrm{q}$

\section{Introduction}

The sensitivity of semiconductor based devices is strongly affected by the presence of noise, which sets the lower limit for signal detection in electronic circuits. For this reason, several studies have analysed the electronic noise in devices operating under static and large-signal periodic conditions [1-8]. In a previous paper, we have shown that the total noise power can be reduced up to $25 \%$ by driving the semiconductor with an electric field given by the sum of a first field with a frequency of $500 \mathrm{GHz}$ and a second field at a twice frequency [9]. Furthermore, the noise power appears to be very sensitive to the amplitudes of the excitation signals. The possibility to reduce the electronic noise level by tuning the amplitude of the higher frequency field can be fruitfully used for high-order harmonics extraction purposes. In fact, studies about the effects of wave-mixing on harmonic generation in semiconductor bulks have shown that the expected generation of odd harmonics of each field is enriched by the generation of satellite harmonics at mixed frequencies and that it is possible to obtain a rich comb-like side band structure by simply tuning the two frequencies and amplitudes $[10,11]$. 
In this work, using a Monte Carlo approach, we investigate the role of the frequency of the two mixed oscillating electric fields on the reduction of the hotcarrier noise in an $n$-type low-doped GaAs semiconductor bulk, with the aim to elucidate if this effect has a frequency dependence.

\section{Procedure for the noise calculation}

When the system is driven by a periodic electric field (cyclostationary conditions) of frequency $f$ and amplitude $E_{1}$ of the type

$$
E(t)=E_{1} \cos (2 \pi f t),
$$

the velocity autocorrelation function $C_{\delta \nu \delta \nu}(t, \tau)$ can be represented as a function of two relevant times [8] as

$$
C_{\delta \nu \delta \nu}(t, \tau)=\left\langle\nu\left(t-\frac{\tau}{2}\right) \nu\left(t+\frac{\tau}{2}\right)\right\rangle-\left\langle\nu\left(t-\frac{\tau}{2}\right)\right\rangle\left\langle\nu\left(t+\frac{\tau}{2}\right)\right\rangle,
$$

where $\tau$ is the correlation time, and the average is over the sequence of equivalent time moments $t=s+m T_{\mathrm{L}}$, with $s$ belonging to the time interval $\left[0, T_{\mathrm{L}}\right]$ and $m$ is an integer [8]. This two-time symmetric correlation function excludes any regular contribution by describing only the fluctuating part of $\nu(t)$. By averaging over the whole set of values of $t$ during the period $T_{\mathrm{L}}$, the velocity autocorrelation function is obtained as

$$
C_{\delta \nu \delta \nu}(\tau)=\frac{1}{T_{\mathrm{L}}} \int_{0}^{T_{\mathrm{L}}} C_{\delta \nu \delta \nu}(t, \tau) \mathrm{d} t .
$$

The spectral density of the velocity fluctuations is related to the autocorrelation function through the well-known Wiener-Kintchine theorem

$$
S_{\delta \nu \delta \nu}(\nu)=\int_{-\infty}^{\infty} C_{\delta \nu \delta \nu}(\tau) \mathrm{e}^{\mathrm{i} 2 \pi \nu \tau} \mathrm{d} \tau
$$

In this paper, we concentrate our analysis to the case of a GaAs semiconductor bulk driven by a periodic electric field $E(t)$ given by the sum of two parallel fields of amplitudes $E_{1}$ and $E_{2}$ and frequencies $f_{1}$ and $f_{2}$, respectively

$$
E(t)=E_{1} \cos \left(2 \pi f_{1} t\right)+E_{2} \cos \left(2 \pi f_{2} t\right)
$$

We assume the frequency $f_{2}$ to be twice the frequency $f_{1}$. Consequently, in the computation of the velocity autocorrelation function, we can take the period of the lowest frequency excitation field as the time interval $T_{\mathrm{L}}$ delimiting the sequence of equivalent time moments and we can average over the same sequence of equivalent time moments $t=s+m T_{\mathrm{L}}$, for both the fields.

\section{Numerical results and conclusions}

In this work, the Monte Carlo simulations of the motion of electrons in the $n$-type GaAs semiconductor bulk driven by an alternating electric field are computed by following the standard procedure described in Ref. [12]. The conduction bands of GaAs are: the $\Gamma$ valley, four equivalent $L$-valleys and three equivalent $X$-valleys. We take into account the non-parabolicity of the band structure and 
the intervalley and intravalley scattering of electrons in multiple energy valleys. The parameters of the $n$-GaAs band structure and scattering mechanisms used in MC simulation are also taken from Ref. [12]. We assume field-independent scattering probabilities; accordingly, the influence of the two external fields is taken into account only indirectly through the field-modified electron velocities. All the results presented in the current paper are obtained by a single-particle dynamics in a stationary regime, after a transient time of a few ps has elapsed. All simulations are computed by adopting a free electron concentration $n=10^{13} \mathrm{~cm}^{-3}$ and a lattice temperature $T=80 \mathrm{~K}$. We assume that all donors are ionised and that the free electron concentration is equal to the doping concentration. In the calculation of the autocorrelation function we consider $10^{3}$ possible initial values of $s$ and about $10^{6}$ total number of equivalent time moments $m$.

In order to clarify the role of the frequency in the suppression of the electronic noise, we have investigated how the reduction effect of the spectral density observed at $500 \mathrm{GHz}$ in Ref. [9] is modified by working at different frequencies. For this reason we have adopted the following set of conditions for the first field: $E_{1}=$ $12.5 \mathrm{kV} / \mathrm{cm}$ at $f_{1}=200 \mathrm{GHz}, E_{1}=13 \mathrm{kV} / \mathrm{cm}$ at $f_{1}=300 \mathrm{GHz}, E_{1}=14 \mathrm{kV} / \mathrm{cm}$ at $f_{1}=400 \mathrm{GHz}$ and $E_{1}=15 \mathrm{kV} / \mathrm{cm}$ at $f_{1}=500 \mathrm{GHz}$. These amplitudes and frequencies have been chosen in order to have a maximum in the integrated spectral density of the velocity fluctuations. We always consider $f_{2}=2 f_{1}$ and change the amplitude $E_{2}$ in the range $5 \div 25 \mathrm{kV} / \mathrm{cm}$. Figure 1 illustrates the spectral density
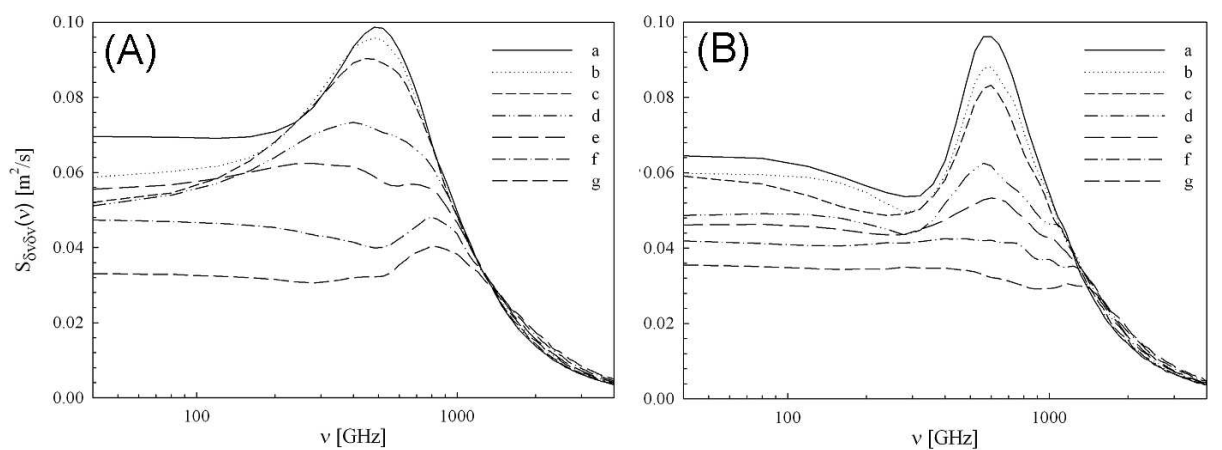

Fig. 1. Spectral density of electron velocity fluctuations as a function of the frequency. Solid lines $(a)$ are used to describe the single field case. In the part (A): $E_{1}=12.5 \mathrm{kV} / \mathrm{cm}$ with $f_{1}=200 \mathrm{GHz}$; in the part (B): $E_{1}=15 \mathrm{kV} / \mathrm{cm}$ with $f_{1}=500 \mathrm{GHz}$. The notsolid lines stand for: (b) $E_{2}=5 \mathrm{kV} / \mathrm{cm} ;(c) E_{2}=7 \mathrm{kV} / \mathrm{cm} ;(d) E_{2}=12 \mathrm{kV} / \mathrm{cm}$; (e) $E_{2}=15 \mathrm{kV} / \mathrm{cm} ;(f) E_{2}=20 \mathrm{kV} / \mathrm{cm} ;(g) E_{2}=25 \mathrm{kV} / \mathrm{cm}$.

obtained by setting $E_{1}=12.5 \mathrm{kV} / \mathrm{cm}$ with $f_{1}=200 \mathrm{GHz}$ in the (A) panel and $E_{1}=15 \mathrm{kV} / \mathrm{cm}$ with $f_{1}=500 \mathrm{GHz}$ in the (B) panel, for different values of the amplitude $E_{2}$. Solid lines are used to describe the case when only the first field is present. For relatively low amplitudes $E_{2}$ the peak is hot-carrier-like and its 
height decreases with the increase in the field $E_{2}$. For stronger fields the peak tends to disappear and the spectral density shows a Lorentzian shape. All these behaviours can be explained by a change in the occupation number of the $\Gamma$ and $L$ valleys due to the effective field experienced by the electrons. The reduction of the hot-carrier peak is related to the transfer of almost all the carriers to the upper energy valley. At $f_{1}=200 \mathrm{GHz}$, for very intense fields $\left(E_{2}>E_{1}\right)$, the spectral density shows once more a hot-carrier peak probably due to the contribution of the partition noise caused by the population of the $X$ valleys.

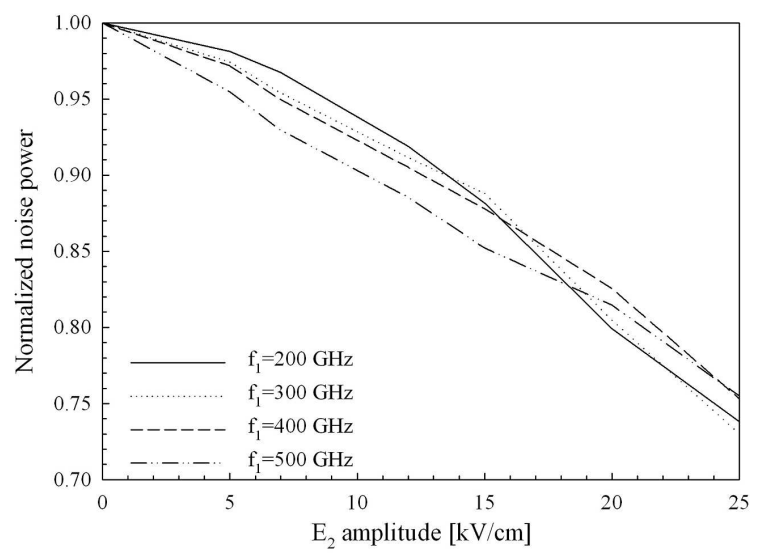

Fig. 2. Total noise power, normalised to the value obtained when only the first field is present, as a function of the amplitude of the second field $E_{2}$ for different frequencies of the applied fields. In all curves $f_{2}=2 f_{1}$.

Figure 2 shows the integrated spectral density (normalised to the value obtained when the second field is absent) as a function of the amplitude $E_{2}$, for $f_{1}=200,300,400$, and $500 \mathrm{GHz}$. In all the studied cases the total noise power is reduced proportionally to the second field amplitude up to a maximum of $25 \%$. The reduction trend is independent of the frequency and nearly linear with the increase in the amplitude $E_{2}$. The circumstance that the reduction is almost independent of the frequency is very interesting because of the dominant role played by the frequency of the mixed fields in the enhancement of the harmonic emission. In fact, by suitably choosing the characteristic parameters of the two mixed fields it is possible to obtain a maximum in the harmonic generation efficiency and a minimum in the electronic noise level.

\section{References}

[1] L. Varani, L. Reggiani, T. Kuhn, T. Gonzalez, D. Pardo, IEEE Trans. Electron Dev. 41, 1916 (1994).

[2] J.-P. Nougier, IEEE Trans. Electron Dev. 41, 2034 (1994). 
[3] L. Reggiani, P. Golinelli, L. Varani, T. Gonzalez, D. Pardo, E. Starikov, P. Shiktorov, V. Gružinskis, Microelectron. J. 28, 183 (1997).

[4] P. Shiktorov, E. Starikov, V. Gruzinskis, S. Perez, T. Gonzalez, L. Reggiani, L. Varani, J.C. Vaissiere, Phys. Rev. B 67, 165201 (2003).

[5] P. Shiktorov, E. Starikov, V. Gružinskis, L. Reggiani, L. Varani, J.C. Vaissière, M. Zarcone, D. Persano Adorno, G. Ferrante, in: Noise in Physical Systems and 1/f Fluctuations, Ed. Gijs Bosman, World Scientific, Singapore 2001, p. 677.

[6] P. Shiktorov, E. Starikov, V. Gružinskis, L. Reggiani, L. Varani, J.C. Vaissire, Appl. Phys. Lett. 80, 4759 (2002).

[7] P. Shiktorov, E. Starikov, V. Gružinskis, L. Reggiani, L. Varani, J.C. Vaissire, S. Perez, T. Gonzalez, J. Comput. Electron. 2, 455 (2003).

[8] T. Gonzalez, S. Perez, E. Starikov, P. Shiktorov, V. Gružinskis, L. Reggiani, L. Varani, J.C. Vaissire, Proc. SPIE 5113, 252 (2003).

[9] D. Persano Adorno, M.C. Capizzo, M. Zarcone, submitted to Fluctuation Noise Lett..

[10] D. Persano Adorno, M. Zarcone, G. Ferrante, Phys. Status Solidi C 0, 1488 (2003).

[11] D. Persano Adorno, M. Zarcone, G. Ferrante, Laser Phys. 13, 270 (2003).

[12] D. Persano Adorno, M. Zarcone, G. Ferrante, Laser Phys. 10, 310 (2000). 